Research

Manuscript Number: ALGAL-D-15-00694R1

Title: Parameter sensitivity analysis of a mechanistic model to simulate microalgae growth

Article Type: Full Length Article

Section/Category: Algal Biotechnology

Keywords: Morris screening; mathematical modeling; one-at-time; sensitivity; microalgae; wastewater

Corresponding Author: Prof. Joan Garcia, PhD

Corresponding Author's Institution: Technical University of Catalonia

First Author: Alessandro Solimeno, Ph.D student

Order of Authors: Alessandro Solimeno, Ph.D student; Joan Garcia, PhD; Roger Samsó

Abstract: In this paper, sensitivity analysis is applied to a mechanistic model developed to simulate microalgae growth. The Morris method of Elementary Effects (EES) is applied to evaluate the sensitivity of model outputs with respect to a subset of key input parameters. For an easier interpretation, results were plotted as distributions of elementary effects means and standard deviations for each input parameter. The model outputs were very sensitive with respect to the maximum specific growth rate of microalgae ( $\mu \mathrm{ALG})$. Results of the sensitivity analysis indicate that the transfer of ammonia ( $\mathrm{Ka}, \mathrm{NH} 3$ ) and carbon dioxide (Ka, CO2) have a non-linear relation with nitrogen uptake and carbonate concentrations, respectively. This analysis helped identify the parameters with the greatest impact on simulation outputs. The results indicated that maximum specific growth rate of microalgae ( $\mu \mathrm{ALG}$ ) was the most critical parameter to calibrate properly. 


\section{Tables}

Table 1. List of model outputs.

\begin{tabular}{|c|c|}
\hline Model outputs & Description \\
\hline $\mathbf{X}_{\mathrm{ALG}}$ & $\begin{array}{l}\text { Concentration of microalgae biomass. It increases with growth processes and decreases by } \\
\text { endogenous respiration and inactivation. }\end{array}$ \\
\hline $\mathrm{S}_{\mathrm{NH} 3}+\mathrm{S}_{\mathrm{NH} 4}$ & $\begin{array}{l}\text { Concentration of nitrogen present in the water as ammonium and ammonia. Nitrogen as } \\
\text { ammonium }\left(S_{N H 4}\right) \text { is produced through the processes of endogenous respiration and through } \\
\text { inactivation of microalgae. It is consumed through the growth of microalgae. Nitrogen in } \\
\left.\text { form of ammonia }\left(S_{N H 3}\right) \text { is in chemical equilibrium with ammonium ( } S_{N H 4}\right) \text {. Its } \\
\text { concentration decreases by volatilization to the atmosphere. }\end{array}$ \\
\hline $\mathrm{S}_{\mathrm{NO} 3}$ & Nitrogen available as nitrate. It is consumed by microalgae $\left(X_{A L G}\right)$. \\
\hline $\mathrm{S}_{\mathrm{HCO3}}+\mathrm{S}_{\mathrm{CO} 2}$ & $\begin{array}{l}\text { Concentration of carbon as carbon dioxide and bicarbonate. Carbon as carbon dioxide } \\
\left(S_{\mathrm{CO} 2}\right) \text { is consumed by microalgae and is produced through the processes of endogenous } \\
\text { respiration and inactivation. Carbon as bicarbonate }\left(S_{\mathrm{HCO} 3}\right) \text { is in chemical equilibrium with } \\
\text { carbon dioxide }\left(S_{\mathrm{CO} 2}\right) \text { and carbonate }\left(S_{\mathrm{CO} 3}\right) \text {. }\end{array}$ \\
\hline $\mathbf{S}_{\mathrm{CO} 3}$ & $\begin{array}{l}\text { Carbon in the form of dissolved carbonate. It is in chemical equilibrium with bicarbonate } \\
\left(S_{\mathrm{HCO} 3}\right) \text { and carbon dioxide }\left(\mathrm{S}_{\mathrm{CO} 2}\right) \text {. Carbonate is not used by microalgae as carbon source. }\end{array}$ \\
\hline
\end{tabular}

Table 2. Sensitivity measures of input parameter at $r=10$ for each output variables.

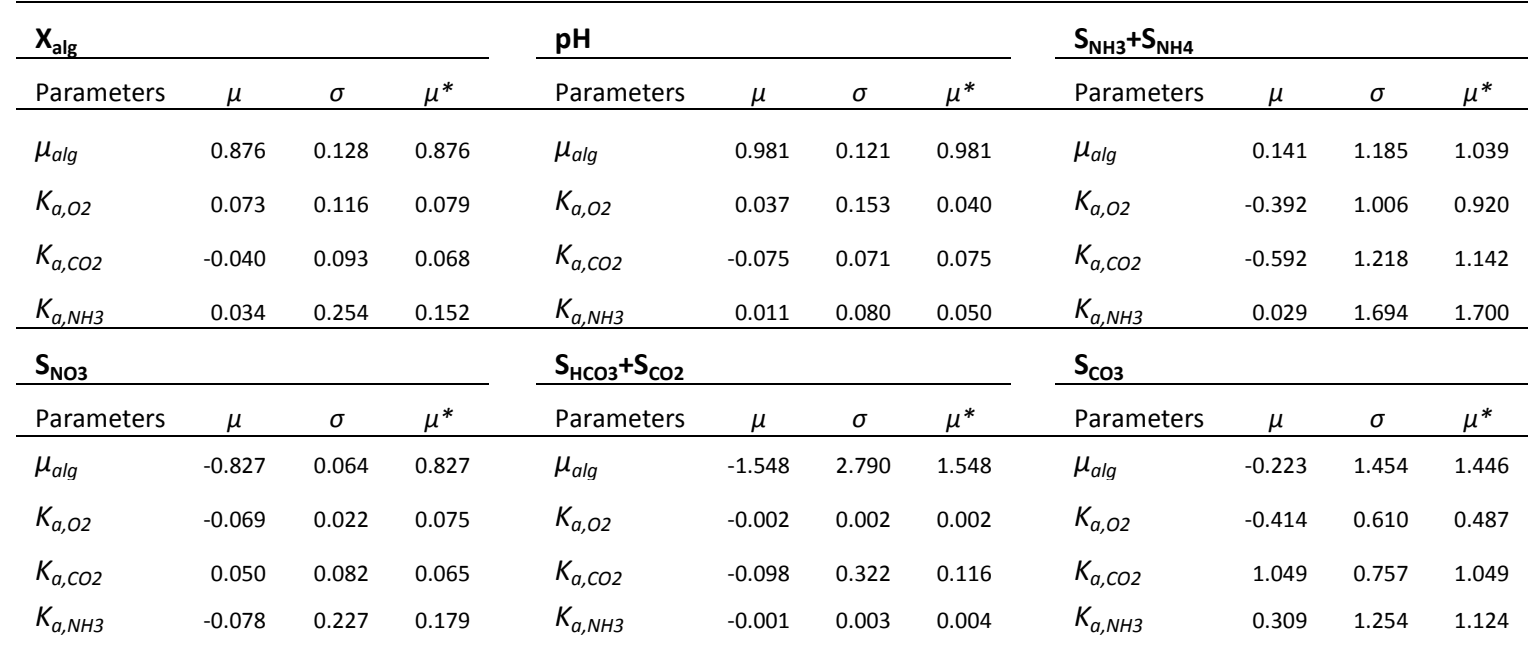




\section{Figures}

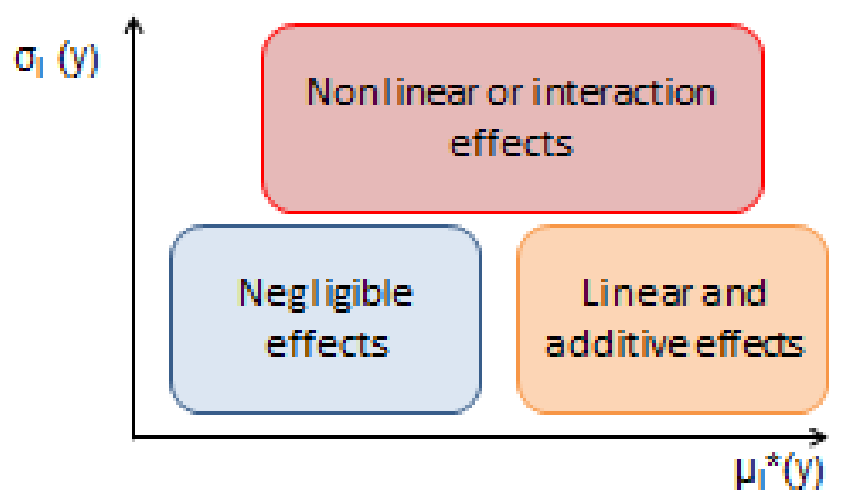

Fig. 1. Schematic representation of theoretical disposition of means $\mu_{i}^{*}$ and standard deviations $\sigma_{i}$ of the effects distribution (Adapted from Santiago et al.,[22]).
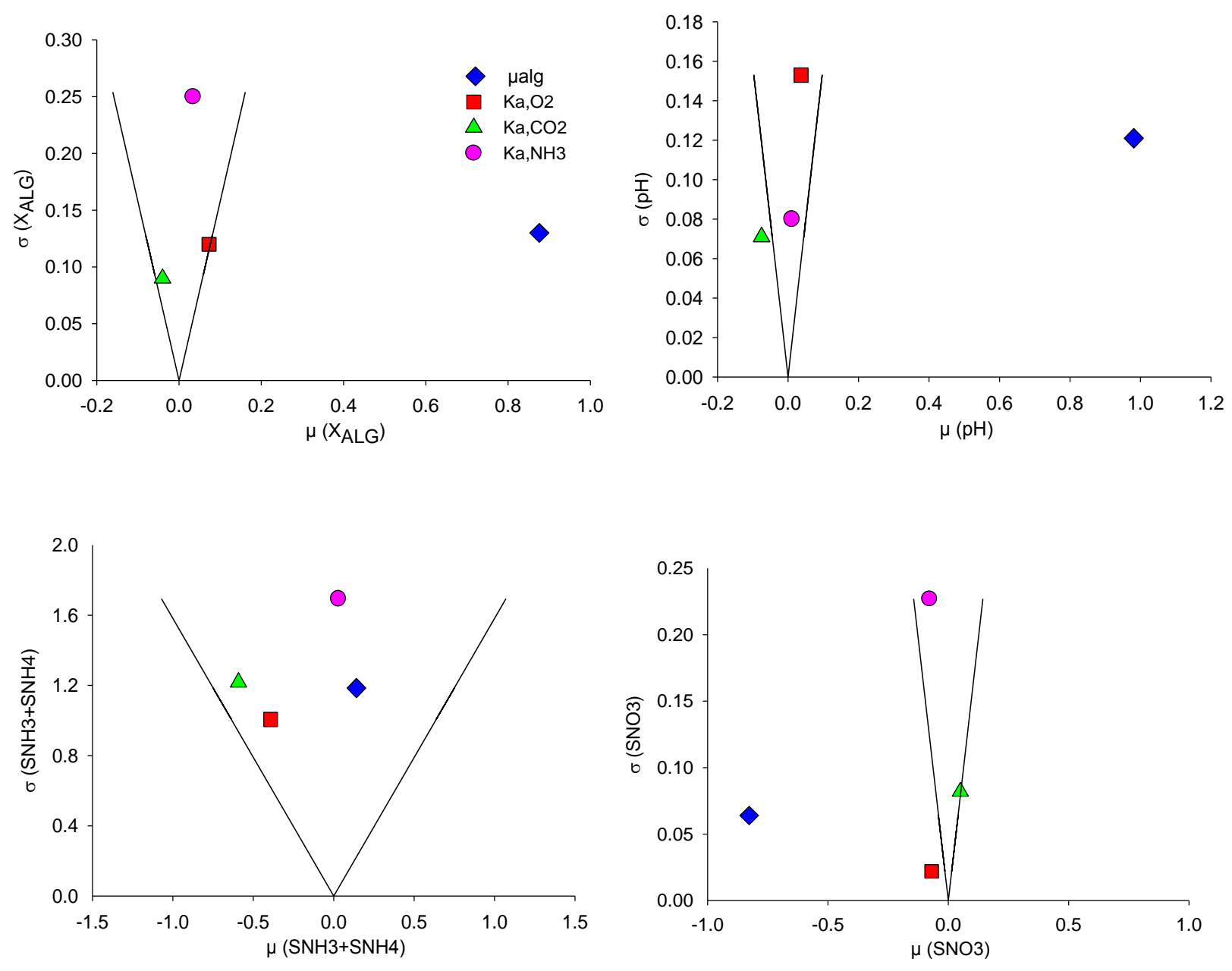

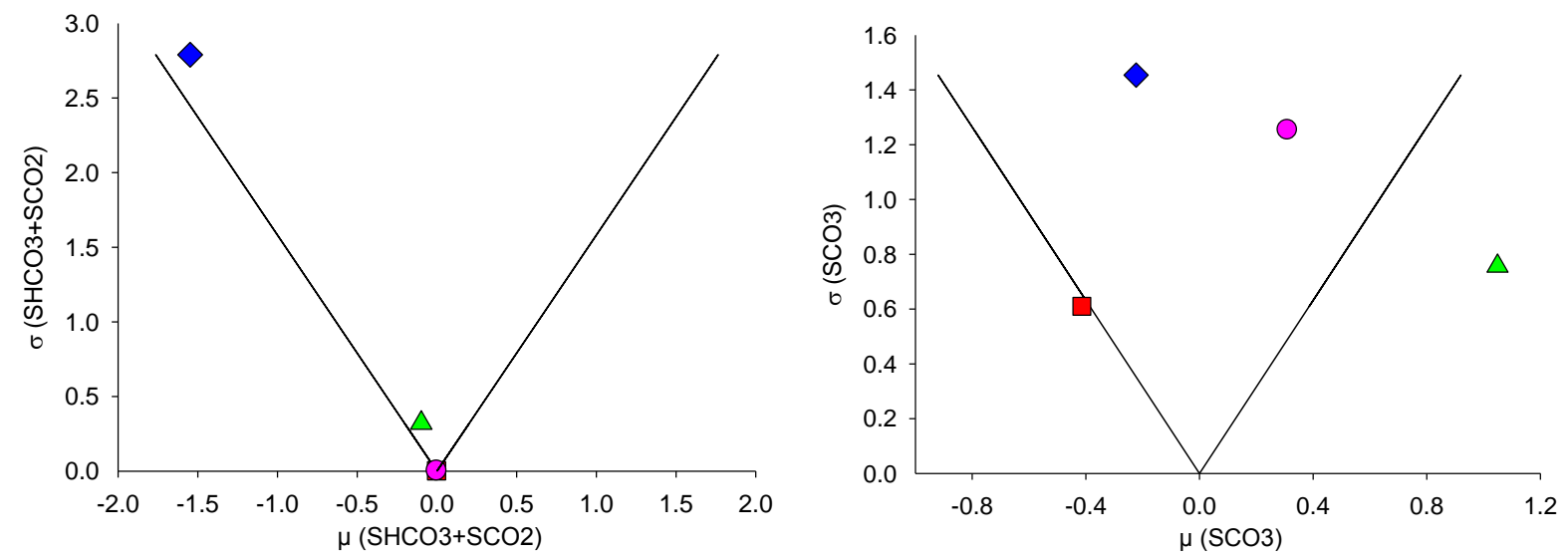

Fig. 2. Sensitivity measures of the distribution of elementary effects of the inputs on the model outputs a) $\mathrm{X}_{\mathrm{alg}}$, b) $\left.\mathrm{pH}, \mathrm{c}\right) \mathrm{S}_{\mathrm{NH} 3}+\mathrm{S}_{\mathrm{NH} 4}$, d) $\mathrm{S}_{\mathrm{NO} 3}$, e) $\mathrm{S}_{\mathrm{HCO} 3}+\mathrm{S}_{\mathrm{CO} 2}$, f) $\mathrm{S}_{\mathrm{CO} 3}$. Lines correspond to $\mu_{i}= \pm 2 S E M_{i}$. Figure legends for graphics shown in the upper right graph.
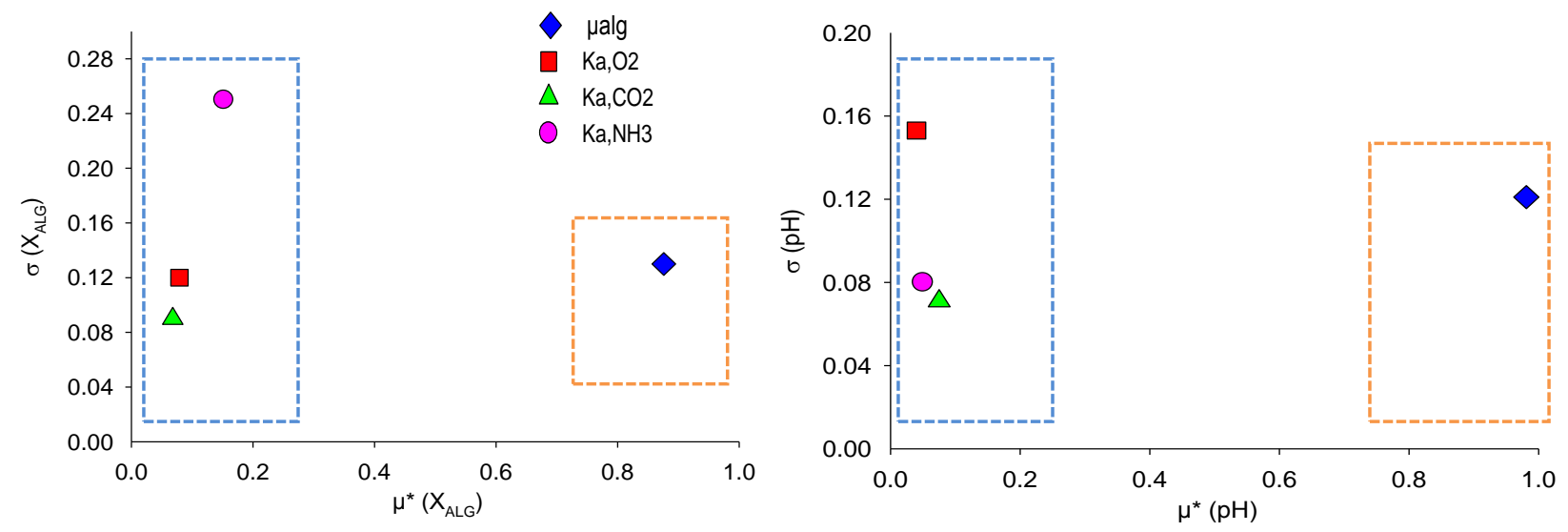

a)

b)
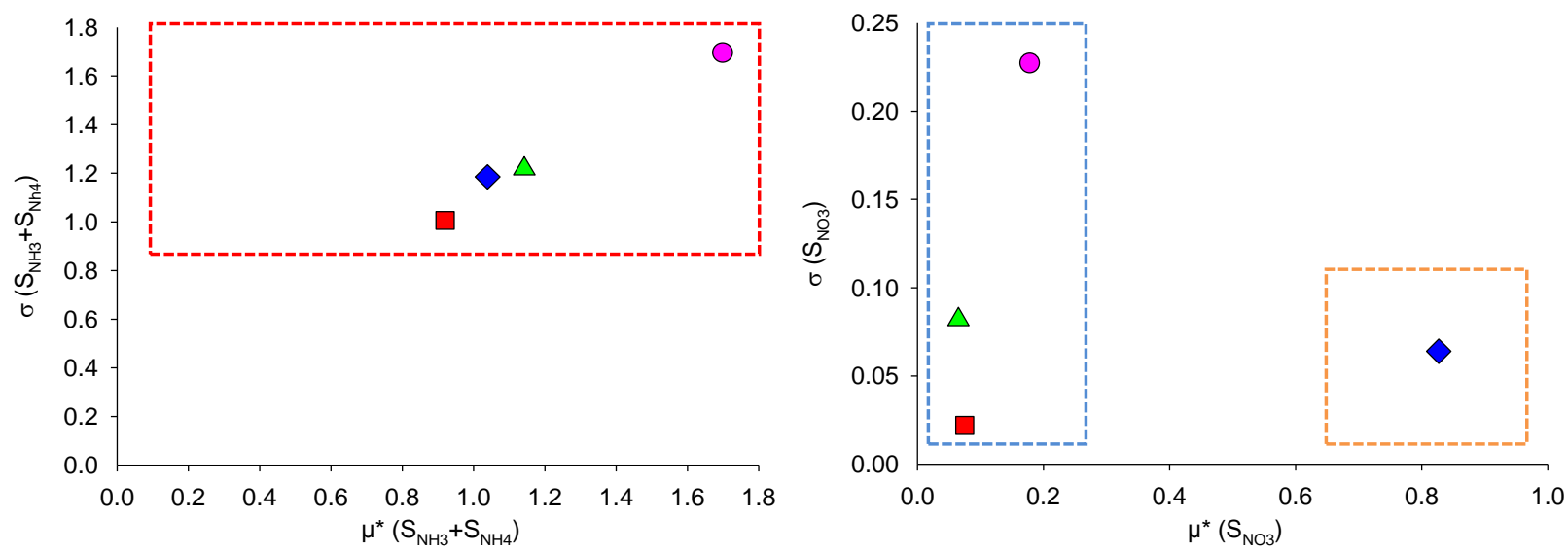

c)

d) 


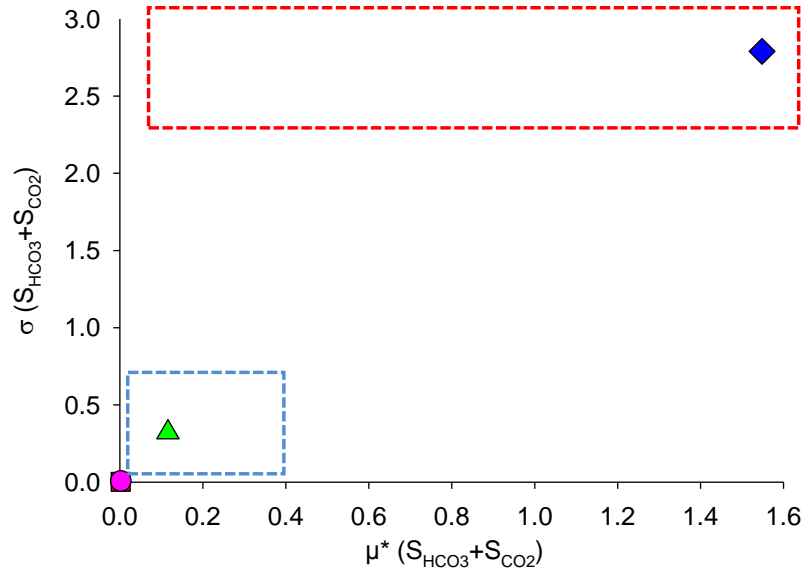

e)

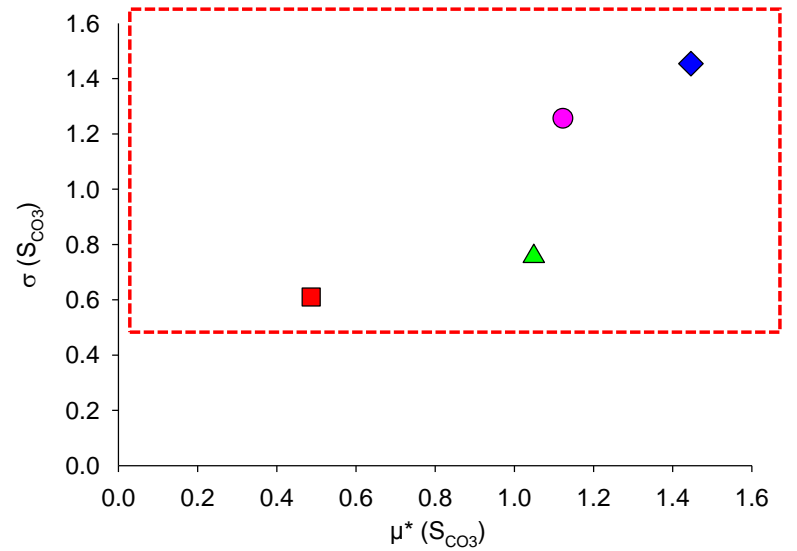

f)

Fig. 3. Sensitivity measures $\mu_{i, j}$ * versus $\sigma_{i, j}$ for the model outputs a) $X_{\text {alg }}$, b) $p H$, c) $\mathrm{S}_{\mathrm{NH} 3}+\mathrm{S}_{\mathrm{NH} 4}$, d) $\mathrm{S}_{\mathrm{NO} 3}$, e) $\mathrm{S}_{\mathrm{HCO} 3}+\mathrm{S}_{\mathrm{CO} 2}$, f) $\mathrm{S}_{\mathrm{CO} 3}$. Dotted lines represent the theoretical distribution of effects: negligible effects (blue dotted line), non-linear effects (red dotted line) and linear effect (orange dotted line). Figure legends for graphics shown in the upper right graph. 
Supplementary Material
Click here to download Supplementary Material: Solimeno et al., Morris Method.xlsx

Supplementary Material
Click here to download Supplementary Material: Solimeno et al., Morris Method.xIsx

cher

(a)

$x$

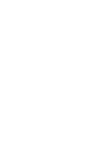

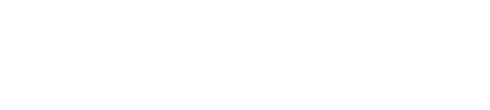

. 
Graphical abstract
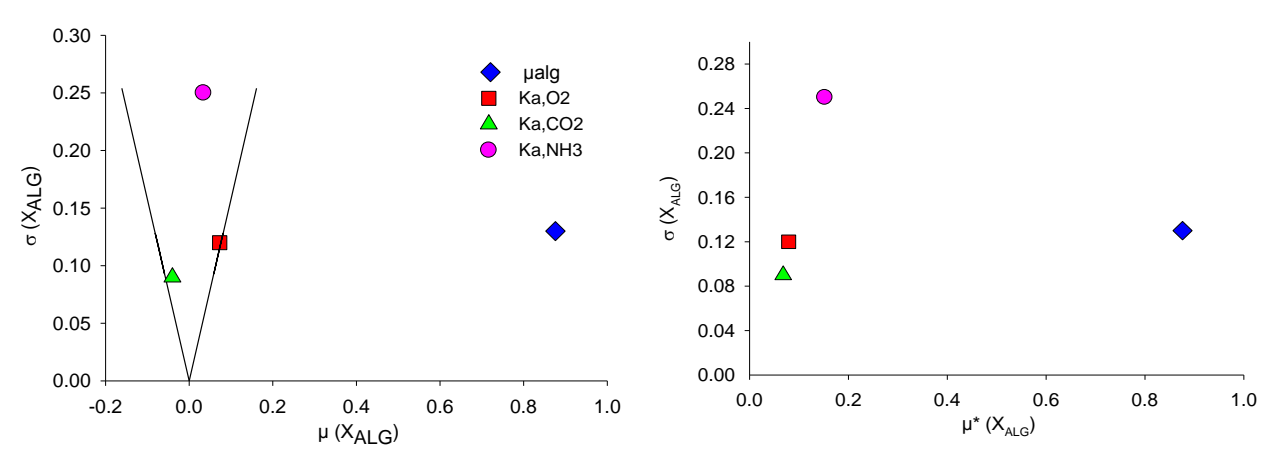

\section{Graphical Abstract}


Highlights

\section{Highlights:}

- Morris's method: procedure and sensitivity measurements.

- Application of Morris's method to the microalgae growth model.

- The maximum specific growth rate of microalgae is the most sensitive parameter. 


\title{
Parameter sensitivity analysis of a mechanistic model to simulate microalgae growth
}

\author{
Alessandro Solimeno*, Roger Samsó** and Joan García* \\ *GEMMA - Group of Environmental Engineering and Microbiology, Department of Hydraulic, Maritime and Environmental \\ Engineering, Universitat Politècnica de Catalunya-BarcelonaTech, c/Jordi Girona, 1-3, Building D1, E-08034, Barcelona, Spain. \\ **IRSTEA - Freshwater Systems, Ecology and Pollution Research Unit, 5 rue de la Doua,CS70077, 69626 Villeurbanne cedex, \\ France. \\ Corresponding author. Tel,: +34 93401 6464; fax 34934017357. \\ E-mail address: joan.garcia@upc.edu (J. García).
}

\begin{abstract}
In this paper, sensitivity analysis is applied to a mechanistic model developed to simulate microalgae growth. The Morris method of Elementary Effects (EEs) is applied to evaluate the sensitivity of model outputs with respect to a subset of key input parameters. For an easier interpretation, results were plotted as distributions of elementary effects means and standard deviations for each input parameter. The model outputs were very sensitive with respect to the maximum specific growth rate of microalgae $\left(\mu_{A L G}\right)$. Results of the sensitivity analysis indicate that the transfer of ammonia $\left(K_{a, \mathrm{NH} 3}\right)$ and carbon dioxide $\left(K_{a, \mathrm{CO} 2}\right)$ have a non-linear relation with nitrogen uptake and carbonate concentrations, respectively. This analysis helped identify the parameters with the greatest impact on simulation outputs. The results indicated that maximum specific growth rate of microalgae $\left(\mu_{A L G}\right)$ was the most critical parameter to calibrate properly.
\end{abstract}

Keywords: Morris screening, mathematical modeling, one-at-time, sensitivity, microalgae, wastewater.

\section{Highlights:}

- Morris's method: procedure and sensitivity measurements.

- Application of Morris's method to the microalgae growth model.

- The maximum specific growth rate of microalgae is the most sensitive parameter.

\section{Graphical abstract}
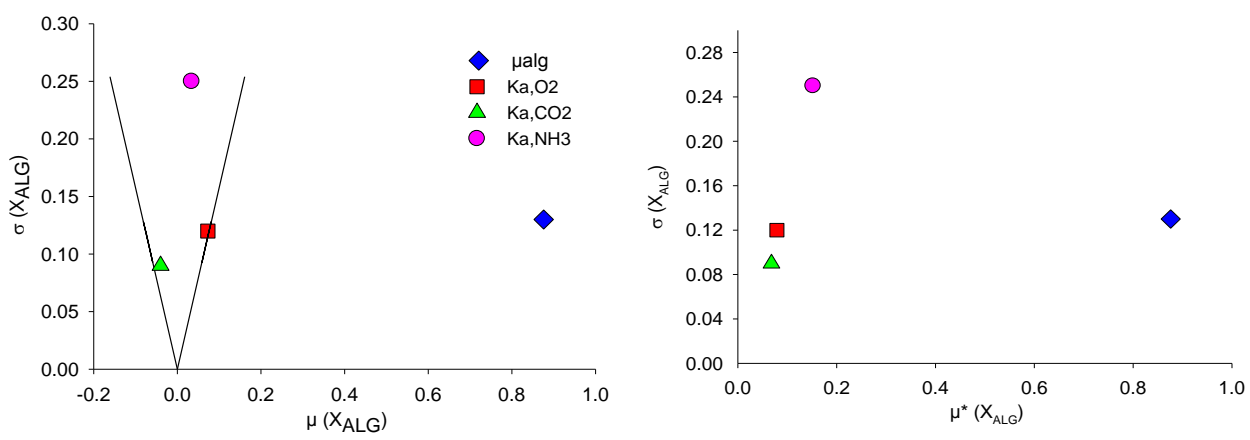


\section{Introduction}

Full-scale microalgae cultures are used to produce a variety of compounds for different economic sectors such as: aquaculture and animal feed; human nutrition; cosmetics and nutraceutics; and pharmaceutics [1,2]. Moreover, mixed cultures of microalgae and bacteria are being used for wastewater treatment in ways that may convert "conventional wastewater treatment plants" into "resource recovery plants", able to produce purified water and by-products such as biodiesel $[3,4]$.

A thorough understanding of the internal functioning of microalgae-based technologies is essential to predict performance and update design guidelines. The physical, chemical, and biological processes that occur in microalgae cultures systems are difficult to study because most of them take place simultaneously and are strongly interdependent. In addition, the rates of many of these processes depend on environmental variables such as light intensity and temperature. In the case of wastewater treatments with mixed cultures, it is very challenging to understand a microbiological system where metabolic processes such as photoautotrophy and heterotrophy coexist.

The increasing number of applications of microalgae-based technologies has encouraged the development of new mathematical models to study the main processes, factors and variables that influence microalgae growth in different types of cultures, including wastewaters. In the last decade, an array of mathematical models that predict microalgae biomass production has been developed [5,6]. One general limitation of these models is the use of very few parameters to describe the inherent complexity of algal cultures, especially in the particular case of microalgae grown in wastewaters, where carbon and/or nitrogen limitation can be significant.

Recently, a complex mechanistic model to simulate microalgae growth in various cultures was developed [7]. This model is a part of a more ambitious project through which we intend to develop a complete model to simulate mixed cultures of microalgae and bacteria treating wastewater (e.g. high rate algal ponds). Therefore, in this first version of the model, only microalgal processes were included, while bacterial processes were not taken into account.

River Water Quality Model 1 (RWQM1) of the International Water Association [8] was used as a reference for the new model. Carbon-limited microalgae growth, transfer of gases to the atmosphere and photorespiration, photosynthesis kinetics and photoinhibition were not included in RWQM1, but were considered as candidate parameters for new model. Furthermore, we felt that growth of microalgae would be dependent on light intensity, temperature, and availability of nitrogen and carbon species.

The model was calibrated using experimental data from a case study based on the cultivation of different microalgae species in a culture medium simulating treated urban wastewater (secondary effluent).

Sensitivity analysis is an important step during model development, promotes better understanding of the complex interactions of engineered systems [9], and can be an important tool for building a the mechanistic model for microalgae growth. With 
this in mind, the aim of the present study was to identify the parameters that have the greatest impact on a new model for microbial culture. Sensitivity analysis of whole set of model parameters (31) is quite an unattainable objective unless high-end computational facilities are available. For this reason, a subset of the most influential parameters on output model was analysed. These subset parameters were selected because they turned out the parameters that most influenced the results obtained with the model and are therefore likely to be changed during calibration.

The Morris method of Elementary Effects (EEs) [10] was selected over other commonly used global sensitivity analysis methods [11] based on previous work by Ruano et al. [12] for screening the most influential parameters in wastewater treatment plant models. The Morris method corresponds to a typically randomized One-At-a-Time (OAT) approach. OAT designs are an efficient technique in which the factors are varied individually by the same relative amount around the nominal point [13]. The basic idea is to reproduce individually randomized experiments that evaluate the elementary effects along trajectories obtained by changing one parameter at a time.

The work described here was necessary to complete the model of Solimeno et al. [7]. Little information was available for several additional parameters related to microbial growth that were thought to be necessary for development of this model.

After model calibration was optimized, the sensitivity analysis described here promoted interpretation of model outputs, and refined our understanding of which parameters were required. As a result, the model provided new insight into the functioning of microalgae cultures, and promoted investigation of the many factors that may influence microalgae growth.

\section{Material and methods}

\subsection{Theoretical background}

The Elementary Effects method represents an effective screening strategy to identify the most important factors in highly parametrized models [14], and is summarized here.

Here is presented a summary of the method following the explanation by Campolongo et al. [15].

Suppose a general model, the model output $\mathrm{y}=\mathrm{y}(\mathbf{x})$ is a scalar function of $k$ dimensional factors (parameters and input values) constituting a general vector $\mathbf{x}$ that identify an exact point in the experimental domain $\Omega$ of $k$-dimensional factor, which corresponds to an exact value of $y$. The vector $\mathbf{x}=\left\{\mathrm{x}_{1}, \mathrm{x}_{2} \ldots \mathrm{x}_{\mathrm{k}}\right\}$ has $k$ components, $x_{i}$, each of which can be take $p$ level in the set $\{0,1 /(p-1), 2 /(\mathrm{p}-1), 3 /(p-1), \ldots,(p-2) /(p-1), 1\}$. This assume that range of any $k$-dimensional factors has been scaled to the set levels $\{0$, $1 /(p-1), 2 /(\mathrm{p}-1), \ldots 1\}$. The region of experimentation $\Omega$ is thus a $k$-dimensional $p$-level grid.

Morris defines the elementary effect of the $i$ th input parameter at given value of $\mathbf{x} \in \Omega$ [10]:

$$
E E_{i}(\mathbf{x})=\left[y\left(x_{1}, x_{2}, \ldots, x_{i-1}, x_{1+\downarrow}, x_{i+1}, \ldots, x_{k}\right)-y(\mathbf{x})\right] / \Delta
$$


where $\Delta$ is the magnitude of step length that can be assumed value in the set $\{1 /(p-1), \ldots 1-1 /(p-1)\}$ so that $\mathbf{x}+\Delta$ is still in $\Omega$.

\subsubsection{Trajectory construction}

The basic principle of Morris's method [10] was applied to build $r$ random orientation in the region of experimentation, $\Omega$, constituted by $p$ levels. The magnitude of the experiment step, $\Delta$, is a multiple of $1 /(p-1)$. It will be convenient to restrict attention to the case in which $p$ is even and $\Delta=p /[2(p-1)]$ for more economical design construction [16].

A base value, $\mathbf{x}^{*}$, is randomly chosen from the vector $\mathbf{x}$ values ranging from 0 to $1-\Delta$, so that increasing by $\Delta$ one of the $k$ components, the vector $\mathbf{x}^{(1)}$ that it still in $\Omega$.

After calculating the elementary effect of the $i$ th component of $\mathbf{x}^{(1)}$ following the Eq. 1., $k+1$ new sampling points are selected such that two consecutive points differ in just one component and the elementary effect for each factor are calculated.

The vector so created $\mathbf{x}^{(1)}, \mathbf{x}^{(2)}, \ldots, \mathbf{x}^{(\mathrm{k}+1)}$ define a trajectory in the parameter space, and an orientation matrix $\mathrm{B}^{*}$.

The final trajectory matrix, $\mathrm{B}^{*}$, as given in the following equation is:

$$
\begin{gathered}
\mathrm{B}^{*}=\left(\mathrm{J}_{\mathrm{m}, 1} \mathrm{X}^{*}+\Delta \mathrm{B}^{\prime}\right) \mathrm{P}^{*} \\
\mathrm{~B}^{*}=\left(\mathrm{J}_{\mathrm{m}, 1} \mathrm{X}^{*}+(\Delta / 2)\left[\left(2 \mathrm{~B}-\mathrm{J}_{\mathrm{m}, \mathrm{k}}\right) \mathrm{D}^{*}+\mathrm{J}_{\mathrm{m}, \mathrm{k}}\right]\right) \mathrm{P}^{*}
\end{gathered}
$$

where

- $\mathrm{J}$ is $\left(m^{*} l\right)$ unit matrix;

- $\mathrm{D}^{*}$ is a $k$-dimensional diagonal matrix which the diagonal elements may be take a value of +1 of -1 with the same probability [17].

- $\mathrm{P}^{*}$ is a $k$-dimensional matrix where each column and row contains only single element equal to 1 and the rest 0 's. The random location of the 1 's changes the order that the variables are perturbed, and increases the number of trajectories [17].

To determine the random directions of the trajectory the matrix B' was created:

$$
B^{\prime}=(1 / 2)\left[\left(2 B-J_{m, k}\right) D^{*}+J_{m, k}\right]
$$

where:

- $\mathrm{J}$ is $\left(m^{*} k\right)$ unit matrix with $m=k+1$;

- B is a random $\left(m^{*} k\right)$ lower left triangle unit matrix with two rows that differ in only one element;

The design matrix $\mathrm{X}$ is constructed by changing the base value $\mathbf{x}^{*}$, or the random selected matrices $\mathrm{B}, \mathrm{D}^{*}$ and $\mathrm{P}^{*} r$ times. The total number of simulations $(\mathrm{N})$ needed in the Morris's method is $\mathrm{N}=\mathrm{r}^{*}(\mathrm{k}+1)$.

\subsubsection{Morris's method indices}


To obtain a non-dimensional measure in this study, the scaled elementary effects

$S E E_{i, j}$ proposed by $S$ in et al. [18] were applied. The unscaled elementary effect $E E_{i, j}$ given by Eq. (1) yields an incorrect classification of parameters for the model, especially when model outputs differ by an order of magnitude [18,19]. This condition justifies the use of the scaled elementary effects:

$$
\operatorname{SEE}_{\mathrm{i}, \mathrm{j}}(\mathbf{x}):\left[y_{j}\left(x_{1}, x_{2}, \ldots x_{\mathrm{i}-1}, x_{\mathrm{i}+\Delta}, x_{\mathrm{i}+1}, \ldots, x_{\mathrm{k}}\right)-y_{j}(\mathbf{x})\right] / \Delta * \sigma_{\mathrm{i}} / \sigma_{\mathrm{y}}
$$

where $\sigma_{i}$ and $\sigma_{y}$ are the standard deviations of the parameters $x_{\mathrm{i}}$ and model outputs $y_{j}$. The finite distribution of the $\mathrm{SEE}_{\mathrm{i}, \mathrm{j}}$ due to the $i$ th input variable on $j$ th model output is denoted as $F_{i, j}$.

The method proposed by Morris provides a global sensitivity measure (mean and standard deviation) of the finite distribution of $p^{\mathrm{k}-1}[p-\Delta(p-1]$ elementary effects associated with each input [16]. Each $F_{i, j}$ contains $r$ independent scaled elementary effects built by sampling $\mathbf{x}$ from $\Omega$. The mean $\mu$ Eq. (5) and standard deviation $\sigma$ Eq. (6) of the distribution $F_{i, j}$ provide an approximate global sensitivity measure. Mean and standard deviation carried out information about the impact of the $i$ th input factor on the output $j$ th and the dependence of its sensitivity on the values of other parameters [13].

A high mean, $\mu$, indicates a parameter with an important overall effect on the output. A high standard deviation, $\sigma$, indicates a parameter with a non-linear effect on the output, or one which interacts with other parameters [20]. Campolongo et al. [21] modified the calculation of $\mu$, denoted $\mu^{*}$ Eq. (7), when the distribution $F_{i, j}$ is nonmonotonic.

$$
\mu_{\mathrm{i}}=\frac{\sum_{n=1}^{r} S E E_{n}}{r}
$$

$$
\sigma_{\mathrm{i}}=\sqrt{\frac{1}{r} \sum_{n=1}^{r}\left(S E E_{n^{-}} \mu_{i}\right)^{\wedge} 2}
$$

$$
\mu_{\mathrm{i}}^{*}=\frac{\sum_{n=1}^{r}\left|S E E_{n}\right|}{r}
$$

Based on the values of $\mu_{i} *$ and $\sigma_{i}$, the Morris method identifies factors having: negligible effects, linear and additive effects, or nonlinear or interactions effects [22]. Fig. 1 illustrates this interpretation of the values $\mu_{i}{ }^{*}$ and $\sigma_{i}$.

To identify the most influential parameters, these sensitivity measures were interpreted using the graphical approach proposed by Morris [10]. In this approach, the value of $\mu_{i, j}$ and $\sigma_{i, j}$ obtained for all the $F_{i, j}$ distributions are displayed together with two lines corresponding to $\mu_{i, j}= \pm 2 S E M_{i, j}$, where $S E M_{i, j}$ represents the standard error of the mean that can be estimated as $S E M_{i, j}=\sigma_{i, j} / \sqrt{r}$. Parameters that lie inside the "wedge" created by the two lines are deemed as non-influential or negligible. Parameters that lie outside the wedge have significant effect on the output [10,18]. 
2.2. Parameter selection, additional parameterization, and sensitivity analysis: computational experiment

\subsubsection{Parameter selection}

The mechanistic model developed by the authors includes a total of 31 parameters [7]. The values of 16 parameters were taken from RWQM1 [8]. Because RWQM1 does not include the parameters related to transfer of gases to the atmosphere, temperature, photorespiration, or carbon limitation on microalgae growth; values of these parameters were obtained from other literature [23,24,25,3].

The subset parameters evaluated were: the maximum specific rate of microalgae growth $\left(\mu_{A L G}\right)$ and those related to the transfer of gases to the atmosphere (oxygen: $K_{a, O 2}$, carbon dioxide: $K_{a, C O 2}$ and ammonia: $\left.K_{a, N H 3}\right)$. The effects of these parameters were investigated respect to the model outputs (Table 1). Note that these four parameters were selected because a global sensitivity analysis of whole set of model parameters (31) is quite an unattainable objective unless high-end computational facilities are available. These four demonstrated to be the parameters that most influenced the results obtained with the model and are therefore likely to be changed during calibration [7].

The global sensitivity analysis was carried out using the same initial conditions, parameters value and geometry (Solimeno et al. [7]).

\subsubsection{Implementation of the Morris`s method}

The software used for the sensitivity analysis was COMSOL Multiphysics ${ }^{\mathrm{TM}}$ v4.3b. As noted above, the total number of simulations (N) needed in the Morris's method is $\mathrm{N}=\mathrm{r}^{*}(\mathrm{k}+1)$, and previous studies have demonstrated that using $p=4$ levels and $r=10$ produces satisfactory results [15]. Therefore, we used $k=4$ uncertain parameters for the screening, and $r=10$ repetitions of elementary effects to obtain a good balance between computational cost and results robustness. Thus, fifty-five simulations were required. Processing time was determined to be 16 seconds per simulation (PC computer, 3.4 GHz Intel Core i7_3770 processor).

The elementary effects were calculated using Eq. 4, which provides random observations of the distribution function $F_{i, j}$.

The parameters of the experiment were set to $p=4, \Delta=p /[2(p-1)]=2 / 3$ and $r=$ 10. Four different levels $(p=4)$ for each factor were considered. So, the $p$ values in the set $\{0,1 /(p-1), 2 /(p-1), \ldots ., 1\}$ would be equivalent to $\{0,1 / 3,2 / 3,1\}$ in our experiment.

Following Morris's method, 10 orientation matrices were generated, and the respective elementary effects for 4 different factors per orientation matrix were estimated from the model output.

The first base values $\mathbf{x}^{*}=\{0,1 / 3,0,1 / 3\}$ were randomly selected from the possible combinations of $\mathbf{x}=\{0,1 / 3,2 / 3,1\}$ ranging from 1 to $1-\Delta$. After that the matrices presented in Eq. 2 and 3 were defined: 
252

$$
\mathbf{J}_{(5,4)}=\left(\begin{array}{llll}
1 & 1 & 1 & 1 \\
1 & 1 & 1 & 1 \\
1 & 1 & 1 & 1 \\
1 & 1 & 1 & 1 \\
1 & 1 & 1 & 1
\end{array}\right)
$$

254

255

$$
\mathrm{D}_{(4,4)}=\left(\begin{array}{cccc}
1 & 0 & 0 & 0 \\
0 & -1 & 0 & 0 \\
0 & 0 & 1 & 0 \\
0 & 0 & 0 & -1
\end{array}\right)
$$

256

257

$258 \quad \mathrm{P} *(4,4)=\left(\begin{array}{llll}1 & 0 & 0 & 0 \\ 0 & 0 & 1 & 0 \\ 0 & 1 & 0 & 0 \\ 0 & 0 & 0 & 1\end{array}\right)$

260

261

$262 \quad \mathrm{~B}^{\prime}{ }_{(5,4)}=\left(\begin{array}{llll}0 & 1 & 0 & 1 \\ 1 & 1 & 0 & 1 \\ 1 & 0 & 0 & 1 \\ 1 & 0 & 1 & 1 \\ 1 & 0 & 1 & 0\end{array}\right)$

263

264

265

$266 \Delta \mathrm{B}_{(5,4)}^{\prime}=\left(\begin{array}{cccc}0 & 2 / 3 & 0 & 2 / 3 \\ 2 / 3 & 2 / 3 & 0 & 2 / 3 \\ 2 / 3 & 0 & 0 & 2 / 3 \\ 2 / 3 & 0 & 2 / 3 & 2 / 3 \\ 2 / 3 & 0 & 2 / 3 & 0\end{array}\right)$

B' is then multiplied by $\Delta=2 / 3$ defined earlier, to create the following matrix:

267

268

Matrices D* and $\mathrm{P} *$ define the orientation of trajectory (for $k=4$, there are $2^{4}$

269 different possibilities for $D^{*}$ each one with probability $1 / 16$ and $4 !=24$ possibilities for

270 $\mathrm{P}^{*}$ each one with probability $\left.1 / 24\right)$. Then $\mathrm{B}^{*}$ becomes:

271

272

$$
\left.\mathbf{J}_{(4,1)} \mathbf{x}^{*}+\Delta \mathbf{B}^{\prime}\right)^{*} \mathbf{P}^{*}=\left\{\left(\begin{array}{cccc}
1 / 3 & 0 & 1 / 3 & 0 \\
1 / 3 & 0 & 1 / 3 & 0 \\
1 / 3 & 0 & 1 / 3 & 0 \\
1 / 3 & 0 & 1 / 3 & 0 \\
1 / 3 & 0 & 1 / 3 & 0
\end{array}\right)+\left(\begin{array}{cccc}
0 & 2 / 3 & 0 & 2 / 3 \\
2 / 3 & 2 / 3 & 0 & 2 / 3 \\
2 / 3 & 0 & 0 & 2 / 3 \\
2 / 3 & 0 & 2 / 3 & 2 / 3 \\
2 / 3 & 0 & 2 / 3 & 0
\end{array}\right)\right\} *\left(\begin{array}{llll}
1 & 0 & 0 & 0 \\
0 & 0 & 1 & 0 \\
0 & 1 & 0 & 0 \\
0 & 0 & 0 & 1
\end{array}\right)
$$


Finally, matrix $\mathrm{B}^{*}$ becomes

$$
\mathrm{B}^{*}=\left(\begin{array}{cccc}
1 / 3 & 1 / 3 & 2 / 3 & 2 / 3 \\
1 & 1 / 3 & 2 / 3 & 2 / 3 \\
1 & 1 / 3 & 0 & 2 / 3 \\
1 & 1 & 0 & 2 / 3 \\
1 & 1 & 0 & 0
\end{array}\right)
$$

Each row of $\mathrm{B}^{*}$ design the factorization of $k$ parameters. Applying Eq. (4), an elementary effect will be estimated for each input factor. In order to get an estimation of the distribution of elementary effects for each input factor, the process was repeated $\mathrm{r}=$ 10 times. As a result, the design matrix for the entire experiment becomes:

$$
\mathrm{X}=\left(\begin{array}{c}
B_{1}{ }^{*} \\
B_{2}{ }^{*} \\
\cdots \\
B_{10}{ }^{*}
\end{array}\right)
$$

In supplementary material readers can find an Excel file which contains a simplified numerical example of trajectory construction of Morris method. In this example only 2 trajectories out of the 10 selected in this paper are described to make it easier.

\section{Results}

The Morris's method results were evaluated by comparing the means and standard deviations of the distribution function $F_{i, j}$ for each input. Table 2 shows the resulting sensitivity measures $\left(\mu_{i, j}, \mu_{i, j} *\right.$ and $\left.\sigma_{i, j}\right)$ of input parameters $\left(\mu_{a l g}, K_{a, O 2}, K_{a, \mathrm{CO} 2}\right.$, $\left.K_{a, N H 3}\right)$ for each output variable analysed at $r=10$.

Means and standard deviations of the 4 input parameters were plotted in Fig. 2 for the 6 output variables considered $\left(\mathrm{X}_{\mathrm{ALG}}, \mathrm{pH},\left(\mathrm{S}_{\mathrm{NH} 3}-\mathrm{S}_{\mathrm{NH} 4}\right), \mathrm{S}_{\mathrm{NO} 3},\left(\mathrm{~S}_{\mathrm{HCO} 3}-\mathrm{S}_{\mathrm{CO} 2}\right), \mathrm{S}_{\mathrm{CO} 3}\right)$.

In addition there are two lines corresponding to $\mu_{i, j}= \pm 2 S E M_{i, j}$ to facilitate the interpretation of the results. Parameters that lie inside the wedge obtained by the two lines are deemed as non-influential or negligible. Otherwise, if the parameters lie outside the wedge, it indicates to have significant effect on the output $[10,18]$.

Furthermore, Fig. 3 includes the mean effect measures $\mu_{i, j} *$ and the standard deviations $\sigma_{i, j}$ of the distribution of input parameters on model outputs, and illustrates the linearity and interaction effects of the parameters. 


\section{Discussion}

Despite the mechanistic model includes more than 31 parameters, only the sensitivity related to the maximum specific growth rate of microalgae $\left(\mu_{A L G}\right)$ and the parameters of gas transfer to the atmosphere $\left(K_{a, \mathrm{O} 2}, K_{a, \mathrm{CO} 2}\right.$ and $\left.K_{a, \mathrm{NH} 3}\right)$ were analysed the ranges of those obtained from literature were totally unknown unlike the parameters obtained from RWQM1. Moreover, RWQM1's parameters have already been subjected to sensitivity analyses [26].

From the graphical Morris approach (Fig. 2) it was clear that the maximum specific growth rate of microalgae $\left(\mu_{A L G}\right)$ had the greatest influence on microalgae biomass output ( $\left.\mathrm{X}_{\mathrm{ALG}}\right)$ (Fig. 2-a).

This parameter was distributed outside of the "wedge" formed by $\mu_{i, j}= \pm 2$ $S E M_{i, j}$, indicating that model output was very sensitive to this parameter. Altering this parameter by $+/-60 \%$ caused a change in microalgae concentration of $+/-32 \%$. Nitrate and $\mathrm{pH}$ were also very sensitive to microalgae growth rate.

The model was not very sensitive to the transference of gases to the atmosphere. The majority of these parameters $\left(K_{a, O 2}, K_{a, C O 2}\right.$ and $\left.K_{a, N H 3}\right)$ were distributed inside the wedge formed by $\mu_{i, j}= \pm 2 S E M_{i, j}$, indicating that their effects on model output were negligible (Fig. 2-b, c, d, e). Only the transfer of carbon dioxide $\left(K_{a, \mathrm{CO} 2}\right)$ had a clear effect on carbonate in the model output (Fig. 2-f).

To evaluate with more details the effects of these parameters on model outputs, the values of the sensitivity measures $\mu_{i, j} *$ and $\sigma_{i, j}$ were reported in Fig. 3. Maximum specific growth rate of microalgae $\left(\mu_{A L G}\right)$ was the most sensitive input parameter exhibiting a linear relationship with microalgae $\left(X_{A L G}\right), \mathrm{pH}$ and nitrate $\left(S_{N O 3}\right)$, indicated by high $\mu_{i, j} *$ and low $\sigma_{i, j}$ (Fig. 3-a, b, d). Otherwise, $\mu_{A L G}$ exhibited non-linear effects with nitrogen as ammonium and ammonia, and with (dissolved) carbon species (Fig. 3c, e, f).

It is important to note that these simulation outputs were sensitive to $\mathrm{pH}$, which in turn was influenced by $K_{a, \mathrm{NH} 3}$ and $K_{a, \mathrm{CO} 2}$. Thus the transfer of ammonia $\left(K_{a, \mathrm{NH} 3}\right)$ and carbon dioxide $\left(K_{a, \mathrm{CO} 2}\right)$ presented a non-linear or interaction effect on nitrogen $\left(\mathrm{S}_{\mathrm{NH} 3}+\mathrm{S}_{\mathrm{NH} 4}\right)$ uptake and carbonate concentrations.

The effect of growth rate on $\mathrm{pH}$ and nitrate in the model was mediated through microalgae biomass $\left(\mathrm{X}_{\mathrm{ALG}}\right)$ : growth of microalgae consumes substrates (nitrogen and inorganic carbon) and releases hydroxide ions that increase $\mathrm{pH}$. Similarly, the concentration of nitrate depended exclusively on microalgae uptake, in contrast with ammonia which was also affected by transfer to the atmosphere.

Although parameters related to dissolved carbon were also influenced by values of other parameters (i.e., $K_{a, O 2}, K_{a, \mathrm{CO} 2}$ and $K_{a, N H 3}$ ) through interactions effects, the effects of the transfer of gases to the atmosphere $\left(K_{a, \mathrm{O} 2}, K_{a, \mathrm{CO} 2}\right.$ and $\left.K_{a, \mathrm{NH} 3}\right)$ directly on model outputs were typically negligible. The exceptions to this included transfer of ammonia $\left(K_{a, N H 3}\right)$ and carbon dioxide $\left(K_{a, \mathrm{CO} 2}\right)$ with respect to carbonate and ammonium and ammonia concentrations, respectively; these were characterized by high mean and standard deviations outputs. 
The value $\left(\mu_{A L G}=1.5\left[\mathrm{~d}^{-1}\right]\right)$ used during the calibration of the model was in agreement within literature ranges [0.4-2 $\mathrm{d}^{-1}$ ] [8]. Despite model results obtained during the calibration, the results from sensitivity analysis have shown that the model was not sensitive to the parameters related to the transfer of gases to the atmosphere $\left(K_{a, O 2}\right.$, $K_{a, \mathrm{CO} 2}$ and $\left.K_{a, \mathrm{NH} 3}\right)$. The range of these parameters for OD geometry is not known. Because transfer of gases to atmosphere depends on the dimensions of the air-water interface, we initially applied a range of $144-408 \mathrm{~d}^{-1}$ for 2D geometry [27].

In this case, model outputs were very sensitive to parameters related to transfer of these gases to the atmosphere. Subsequently, we determined an optimal range [0.7-4 $\mathrm{d}^{-1}$ ] for OD geometry during model calibration. However, as a result of the present study, we found that the parameters related to the transfer of gases to the atmosphere may vary $+/-60 \%$ of the optimal range with negligible effect on model outputs.

\section{Conclusions}

A sensitivity analysis of the maximum specific rate of microalgae growth $\left(\mu_{A L G}\right)$ and the parameters related to the transfer of gases to the atmosphere $\left(K_{a, O 2}, K_{a, C O 2}\right.$, $K_{a, N H 3}$ ) was conducted on a mechanistic model developed to simulate microalgae growth in wastewater. The Morris method was used to identify the sensitivity of model outputs to 4 parameters calibrated during model building.

The results of the sensitivity analysis indicated that model outputs were especially sensitive to the maximum specific growth rate of microalgae $\left(\mu_{A L G}\right)$, while the parameters related to transfer of ammonia $\left(K_{a, \mathrm{NH} 3}\right)$ and carbon $\left(K_{a, \mathrm{CO} 2}\right)$ to the atmosphere had a non-linear effect on the nitrogen uptake and carbonate concentrations. Thus, maximum specific growth rate of microalgae $\left(\mu_{A L G}\right)$ must be calibrated with great accuracy. The results of this paper have to be considered as a conceptual exercise that has to be verified experimentally.

\section{Bibliography}

[1] Spolaore, P., Joannis-Cassan, C., Duran, E., Isambert, A. Commercial Applications of Microalgae. Journal of bioscience and bioengineering 2006, 101, 87-96.

[2] Acien , F., Fernández Sevilla, J.M., Molina Grima, E. 2013. Photobioreactors for the production of microalgae. Reviews in Environmental Science and Bio/Technology, Volume 12, Issue 2, pp 131-151.

[3] Chisti, Y., 2007. Biodiesel from microalgae. Biotechnology advances 25(3), 294-306.

[4] Brennan, L., Owende, P. Biofuels from microalgae-A review of technologies for production, processing, and extractions of biofuels and co-products. Renewable and Sustainable Energy Reviews, 14, Issue 2, 2010, 557-577.

[5] Bernard, O., Masci, P., Sciandra, A. A photobioreactor model in nitrogen limited conditions. In: Proceedings of the sixth conference on mathematical model- ling, Vienna, 2009.

[6] Packer, A., Li, Y., Andersen, T., Hu, Q., Kuang, Y., Sommerfeld, M. Growth and neutral lipid synthesis in green microalgae: a mathematical model. Bioresour Technol 2011;102:111-7. 
[8] Reichert, P., Borchardt, D., Henze, M., Rauch, W., Shanahan, P., Somlyódy, L., Vanrolleghem, P., 2001. River Water Quality Model no. 1 (RWQM1): II. Biochemical process equations. Water science and technology : a journal of the International Association on Water Pollution Research 43(5), 11-30.

[9] Sin, G., Gernaey, K.V., Improving the Morris method for sensitivity analysis by scaling elementary effects. 2009. 19th European Symposium on Computer Aided Process Engineering - ESCAPE19 J. Jezowski and J. Thullie (Editors).

[10] Morris, M.D. 1991. Factorial Sampling Plans for Preliminary Computational Experiments. Technometrics, Vol. 33, No. 2. pp. 161-174.

[11] Saltelli, A., S. Tarantola, and F. Campolongo (2000), "Sensitivity analysis as an ingredient of modelling", Statistical Science, 15(4): 377-395.

[12] Ruano, M.V., Ribes, J., Ferrer, J., and Sin, G. Application of the Morris method for screening the influential parameters of fuzzy controllers applied to wastewater treatment plants. 2011. Water Science \& Technology 63.10.

[13] Sumner, T., Shephard, E., Bogle, I.D.L., 2012. A methodology for global-sensitivity analysis of time-dependent outputs in systems biology modelling. J. R. Soc. Interface 9, 2156-2166.

[14] Campolongo, F., Saltelli, A., Cariboni, J. 2011. From screening to quantitative sensitivity analysis. A unified approach. Computer Physics Communications 182 (2011) 978-988.

[15] Campolongo, F., Tarantola, S., Saltelli, A., 1999. Tackling quantitatively large dimensionality problems. Computer Physics Communications 117, 75e85.

[16] Alam, F.M., McNaught, K.R., Ringrose, T.J. 2004. Using Morris' randomized OAT design as a factor screening method for developing simulation metamodels. Proceedings of the 2004 Winter Simulation Conference R .G. Ingalls, M. D. Rossetti, J. S. Smith, and B. A. Peters, eds.

[17] King, D.M., Perera, B.J.C. 2013. Morris method of sensitivity analysis applied to assess the importance of input variables on urban water supply yield - A case study. Journal of Hydrology 477, 17-23.

[18] Sin, G., Gernaey, K.V., Neumann, M.B., van Loosdrecht, M.C.M., Gujer, W., 2009. Uncertainty analysis in WWTP model applications: a critical discussion using an example from design. Water Res. 43 (11), 2894-2906.

[19] Smith, R. C. 2014. Uncertainty Quantification Theory, Implementation, and Applications. SIAM Computational Science and Engineering Series, CS12, 2014.

[20] Campolongo, F., Braddock, R., 1999. The use of graph theory in the sensitivity analysis of the model output: a second order screening method. Reliability Engineering and System Safety $641-12$.

[21] Campolongo, F., Cariboni, J., Saltelli, A. 2007. An effective screening design for sensitivity analysis of large models Environmental Modelling \& Software 22 1509e151.

[22] Santiago, J., Corre, B., Claeys-Bruno, M., Sergent, M. 2012. Improved sensitivity through Morris extension. Chemometrics and Intelligent Laboratory Systems 113 52-57.

[23] Silva, H.J., Pirt, J. 1984. Carbon dioxide inhibition of photosynthetic growth of chlorella. Journal of General Microbiology, 130, 2833-2838.

[24] Novak, J.T., Brune, D.E. 1985. Inorganic carbon limited growth kinetics of some freshwater algae. Water Res. $19,215-225$. 
[25] Dauta, A., Devaux, J., Piquemal, F., Boumnich, L. 1990. Growth rate of four freshwater algae in relation to light and temperatura. Hydrobiologia 207, 221-226.

459

[26] Reichert, P.,Vanrolleghem. P., 2011. Identifiability and uncertainty analysis of the River Water Quality Model No. 1 (RWQM1). Water Science and Technology Vol 43 No 7, pp 329-338. cathodic half cell. Bioresour Technol 2009;100(1): 269-74. 